BULL. AUSTRAL. MATH. SOC.

MOS 10A30, 10F20, 10F10

VOL. $6(1972), 317-318$.

\title{
Simultaneous diophantine approximation
}

\section{J.M. Mack}

Two problems are studied in this thesis. The first one is connected with work of Szekeres on higher dimensional analogues of the continued fraction process. The ordinary continued fraction process is generalized to arbitrary rational lines in $R^{n}$, and the ideas involved are used to investigate the connections between rational dependence and approximation algorithms in $R^{n}$. Such algorithms are considered as generalized Farey dissections, a viewpoint going back to Hurwitz.

The second problem is that of improving the upper bound for the approximation constant $c_{2}$, the infimum of positive numbers $c$ such that the inequality

$$
\max (\|q x\|,\|q y\|)<c q^{-1 / 2}
$$

has solutions in arbitrarily large integers $q$ for all real $x, y$. Using a method suggested by E.S. Barnes, an improvement on an estimate due to Davenport has been obtained.

\section{References}

[1] H. Davenport, "Simultaneous diophantine approximation", Proc. London Math. Soc. (3) 2 (1952), 406-416.

[2] J.M. Mack, "A note on simultaneous approximation", BuZZ. Austral. Math. Soc. 3 (1970), 81-83.

[3] J.M. Mack, "On the continued fraction algorithm", Bull. Austral. Math. Soc. $3(1970), 413-422$.

Received 15 November 1971. Thesis submitted to the University of Sydney, March 1971. Degree approved, October 1971. Supervisor: Professor G. Szekeres. 
[4] G. Szekeres, "Multidimensional continued fractions", Ann. Univ. Sci. Budapest. Eötvös Sect. Math. 13 (1970), 113-140. 